\title{
Spontaneous recovery as a function of fixed ratio ${ }^{1}$
}

MARK J. FISCHER AND MICHAEL COLE UNIVERSITY OF CALIFORNIA, IRVINE

Twenty-four rats were run in a replication and extension of Mowrer \& Jones' (1945) and Boren's (1961) studies of the relation between fixed ratio acquisition and extinction. Greater resistance to extinction and greater spontaneous recovery were obtained for higher fixed ratios.

This investigation is primarily a replication of earlier research by Mowrer \& Jones (1945) and Boren (1961) which showed increasing resistance to extinction as fixed ratios (FRs) of reinforcement were increased. Mowrer and Jones proposed a "response unit" notion in which the unit for measuring resistance to extinction is the same pattern of behavior which obtained reinforcement during acquisition. They found that when measured by this method, resistance to extinction decreases as a function of increasing FRs, a finding they attribute to the increased inhibition accruing to long response chains. Their results were replicated by Boren in 1961 .

Deese $(1961$, p. 70$)$ suggests that one way to test the hypothesis that inhibition increases with the size of the response unit is to provide a spontaneous recovery test following extinction. It is predicted that larger FRs will result in greater recovery because of the greater amount of inhibition that has dissipated following extinction.

The present study provides evidence on the relation between the FR in acquisition, extinction, and spontaneous recovery. Spontaneous recovery has not been studied with respect to fixed ratio responding. The closest analogy is a study by Hartman \& Grant (1962) on classical eyelid conditioning, which indicated that spontaneous recovery is greater for random $50 \%$ than $100 \%$ reinforcement.

Method

Subjects and Apparatus. Twenty-four male SpragueDawley rats served as Ss. At the beginning of the experiment they were about 60 days old, weighing from 279 to $481 \mathrm{~g}$ (average $370.25 \mathrm{~g}$ ).

A Lehigh Valley student operant apparatus was employed. The reinforcer was a single $45 \mathrm{mg}$ Noyes pellet. The testing area was shrouded to eliminate light from outside the testing apparatus; a small light was mounted above the cage and an electric fan mounted within the testing area masked noises from outside. The light and the fan were an integral part of a refrigerator-type container, in which the experimental chamber was placed.

The FR reinforcement schedule was programmed by means of a stepper relay which produced a loud click with every bar depression. Responses were recorded on counters and a cumulative recorder.
Procedure. The Ss were maintained throughout the experiment at approximately $80 \%$ of their ad lib body weights. They were divided into four equal groups of six: CRF (continuous reinforcement), FR 2 (fixed ratio 2:1), FR 5 (fixed ratio 5:1), and FR 10 (fixed ratio 10:1). In an initial session, all groups were trained to bar press under CRF and were given 100 reinforcements. During the second session each $S$ was shaped up to his particular fixed ratio schedule and given daily training trials over a five day period at his assigned ratio. On the first day of training, each $S$ received 50 reinforcements, and on the following days, 20 reinforcements. After the five days of training, a single extinction session was run.

At the start of the extinction session, each $S$ was given 10 reinforced trials at his particular fixed ratio. Following this "priming" procedure, each $\mathrm{S}$ was extinguished to a criterion of five or less responses over a 3 min period. After this extinction session, each animal was returned to his home cage for $72 \mathrm{~h}$. At the end of the $72 \mathrm{~h}$ interval (during which time $S$ was maintained on the previous deprivation schedule), $\mathrm{S}$ was returned to the testing apparatus for a spontaneous recovery session. The number of responses per min was recorded over a 20 min period. Results and Discussion

As a measure of asymptotic responding for the four experimental groups, the rate of responding to obtain the 10 reinforcements at the start of the extinction session (responses/min) was calculated for each S. The group means were: 11.5 (CRF), 25.1 (FR 2), 40.6 (FR 5), 34.6 (FR 10). The difference between groups is highly significant $(F=17.3$, df $=$ $3 / 20, p<.01)$. The apparent decrease in rate from FR 5 to FR 10 is not significant ( $F<1$ ). Except for the generally higher rates of responding and the leveling of the function at FR 5-FR 10, these findings are in general agreement with those of Boren (1961).

Figure 1 shows the relation between acquisition FR and extinction performance. The upper curve represents the average trials to the extinction criterion for the four groups. A one-way analysis of variance supports the visual evidence of increasing resistance to extinction for higher FRs $(F=17.4$, $\mathrm{df}=3 / 20, \mathrm{p}<.01$ ). The lower curve was derived from the upper by dividing each group's total trials to extinction by its fixed ratio (i.e., the score for FR 5 is divided by 5) following the procedure first used by Mowrer \& Jones (1945). A clearly decreasing function results $(F=5.9, d f=3 / 20, p<.01)$. 


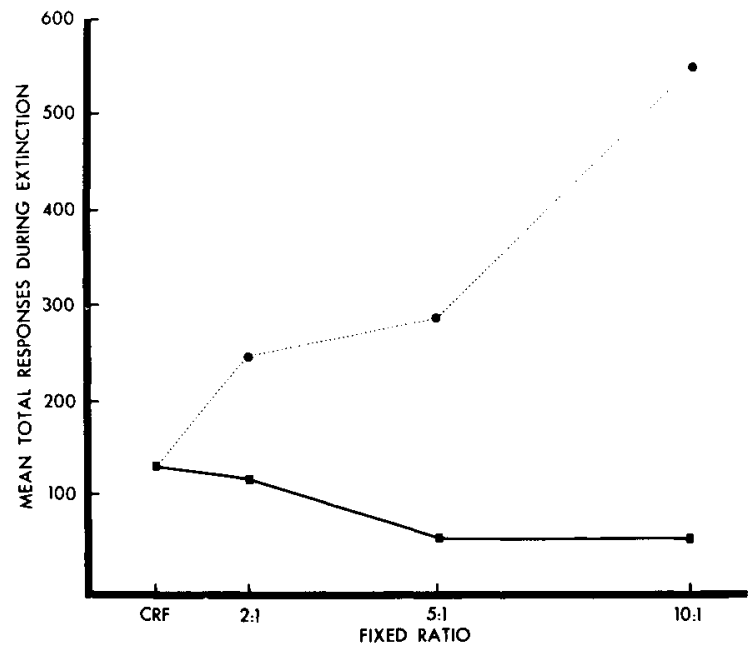

Fig. 1. Mean total response during plotted as a function of acqui sition FR. The solid line is derived from the dotted line by dividing each S's total responses by his FR.

These extinction results represent a very close replication of the findings of Mowrer \& Jones (1945) and Boren (1961).

Figure 2 shows the data for spontaneous recovery plotted in a manner strictly analogous to that used in Fig. 1. The results parallel quite closely the extinction data: Total responding during the spontaneous recovery session is generally greater for the higher FRs $(F=3.4, d f=3 / 20, p<.05)$. The same trends result if only the first $5 \mathrm{~min}$ of the spontaneous recovery session are considered $(F=5.86, \mathrm{df}=3 / 20$, $\mathrm{p}<.01$ ). However, a decreasing function results when the function is plotted in terms of response units ( $F=8.3$, df $=3 / 20, p<.01$ ).

Taken as a whole, these data support the contention that both responding in extinction and spontaneous recovery are increasing functions of acquisition FR. This raises the question of the degree to which responding at each stage of training can be accounted for by characteristics of responding in the previous stage; e.g., is the level of responding in extinction predictable from the level of responding during reinforced training, and is spontaneous recovery performance predictable on the basis of extinction performance? This question was answered by using a performance measure in each stage as the covariate for an analysis of covariance on the succeeding stage.

The first such analysis used the rates of responding to obtain the 10 pellets at the start of the extinction session as a covariate in the analysis of total responses to extinction. Although some of the variance

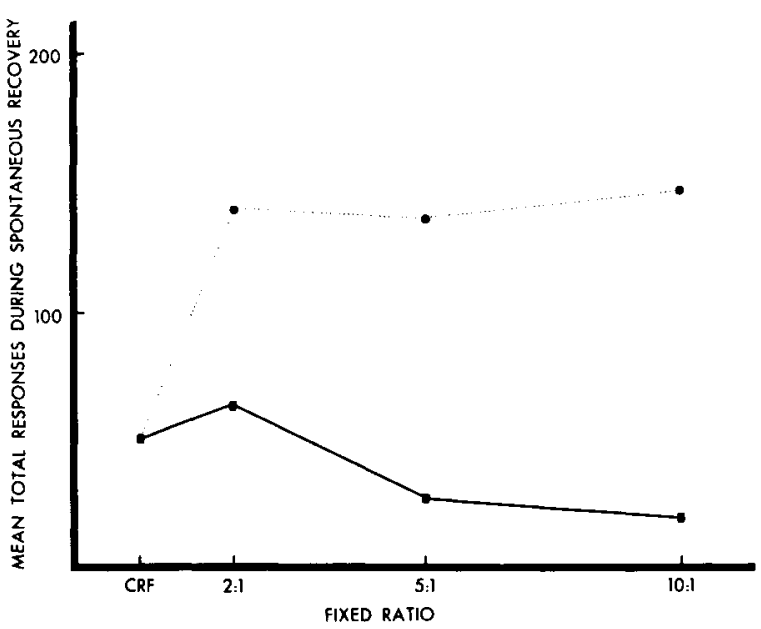

Fig. 2. Mean total responding during spontaneous recovery as a function of FR. The solid line is derived from the dotted line by di viding each S's total responses by his FR.

in extinction performance can be accounted for by terminal acquisition rates, the resulting $F$ is still highly significant ( $F=12.4$, df $=3 / 19, p<.01$ )

In the case of spontaneous recovery, however, performance can be accounted for on the basis of total responses emitted during the extinction session $(F=1.6, d f=3 / 19, p<.10)$.

This disappearance of a significant FR effect after covariance adjustment for extinction performance indicates that nothing new is happening in spontaneous recovery; rather, performance is simply a continuation of the effects already in evidence during extinction. These latter effects, in turn, are a function of acquisition FR.

It does not seem that positing an "inhibitory" process helps in any way to account for these findings, although a reactive inhibition hypothesis is consistent with the data. Fantino (personal communication) suggests that a very high FR would be expected to produce a great deal of inhibition but a decrease in response rate. If it also produced a large spontaneous recovery, support would be gained for the inhibition theory.

\section{References}

BOREN, J. J. Resistance to extinction as a function of the fixed ratio. J. exp. Psychol, 1961, 61, 304-308.

DEESE, J. The psychology of learning. New York: McGraw-Hill, 1961. HARTMAN, T. F., \& GRANT, D. A. Effects of pattern of reinforcement and verbal information on acquisition, extinction and spontaneous recovery of the eyelid CR. J. exp. Psychol, 1962, 63, 217-226. MOWRER, O. H., \& JONES, H. M. Habit strength as a function of the pattern of reinforcement. J. exp. Psychol, 1945, 35, 293-311. Note

1. Reprints available from Michael Cole. 